\title{
Surfaces with constant extrinsically Gaussian curvature in the Heisenberg group
}

\author{
Lakehal Belarbi \\ Department of Mathematics, \\ Laboratory of Pure and Applied Mathematics, \\ University of Mostaganem (U.M.A.B.), Mostaganem, Algeria \\ lakehalbelarbi@gmail.com \\ Submitted: September 19, 2017 \\ Accepted: January 23, 2019 \\ Published online: February 11, 2019
}

\begin{abstract}
In this work we study constant extrinsically Gaussian curvature translation surfaces in the 3-dimensional Heisenberg group which are invariant under the 1-parameter groups of isometries.

Keywords: Constant extrinsically Gaussian curvature Surfaces, Homogeneous group.
\end{abstract}

MSC: 49Q20 53C22.

\section{Introduction}

In 1982, W. P. Thurston formulated a geometric conjecture for three dimensional manifolds, namely every compact orientable 3-manifold admits a canonical decomposition into pieces, each of them having a canonical geometric structure from the following eight maximal and simply connected homogenous Riemannian spaces: $\mathbb{E}^{3}, \mathbb{S}^{3}, \mathbb{H}^{3}, \mathbb{S}^{2} \times \mathbb{R}, \mathbb{H}^{2} \times \mathbb{R}, S L(2, \mathbb{R}), \mathbb{H}_{3}$ and $S_{0}$. See e.g. [34].

During the recent years, there has been a rapidly growing interest in the geometry of surfaces in three homogenous spaces focusing on flat and constant Gaussian curvature surfaces. Many works are studying the geometry of surfaces in homogeneous 3-manifolds. See for example [2-4, 9, 12, 14-16, 21, 22, 24, 36].

The concept of translation surfaces in $\mathbb{R}^{3}$ can be generalized the surfaces in the three dimensional Lie group, in particular, homogeneous manifolds. In Euclidean 
3 -space, every cylinder is flat. Conversely, complete flat surfaces in $\mathbb{E}^{3}$ are cylinders over complete curves. See [20]. López and Munteanu [17] studied invariant surfaces with constant mean curvature and constant Gaussian curvature in $\mathrm{Sol}_{3}$ space. Yoon and Lee [37] studied translation surfaces in Heisenberg group $\mathbb{H}_{3}$ whose position vector $x$ satisfies the equation $\Delta x=A x$, where $\Delta$ is the Laplacian operator of the surface and $A$ is a $3 \times 3$-real matrix.

Flat $G_{4}$-invariant surfaces are nothing but surfaces invariant under $S O(2)$ action, i.e. rotational surfaces. Flat rotational surfaces are classified by Caddeo, Piu and Ratto in [8].

In [14], J. I. Inoguchi give a classification of intrinsically flat $G_{1}$-invariant translation surfaces in Heisenberg group $\mathbb{H}_{3}$. Let $M$ be a surface invariant under $G_{3}$, then $M$ is locally expressed as

$$
X(u, v)=(0,0, v) \cdot(x(u), y(u), 0)=(x(u), y(u), v), u \in I, v \in \mathbb{R} .
$$

Here $I$ is an open interval and $u$ is the arclength parameter. Note that $(x, y, 0)$ and $(0,0, v)$ commute. Then the sectional curvature $K\left(X_{x} \wedge X_{y}\right)=\frac{1}{4}$ and the extrinsically Gaussian curvature $K_{\text {ext }}=-\frac{1}{4}$. Direct computation show that $M$ is flat. (cf. [12-14, 28]).

The paper is divided according the type of surfaces invariant under 1-parameter subgroups of isometries $\left\{G_{i}\right\}_{i=1,2,3,4}$. So, in section 3 we classify $G_{1}$-invariant surfaces of the Heisenberg group $\mathbb{H}_{3}$ with constant extrinsically Gaussian curvature $K_{\text {ext }}$, including extrinsically flat $G_{1}$-invarinant surfaces.

In section 4 we classify $G_{2}$-invariant surfaces of the Heisenberg group $\mathbb{H}_{3}$ with constant extrinsically Gaussian curvature $K_{\text {ext }}$, including extrinsically flat $G_{2^{-}}$ invariant surfaces.

\section{Preliminaries}

The 3-dimensional Heisenberg group $\mathbb{H}_{3}$ is the simply connected and connected 2-step nilpotent Lie group. Which has the following standard representation in $G L(3, \mathbb{R})$

$$
\left(\begin{array}{lll}
1 & r & t \\
0 & 1 & s \\
0 & 0 & 1
\end{array}\right)
$$

with $r, s, t \in \mathbb{R}$. The Lie algebra $\mathfrak{h}_{3}$ of $\mathbb{H}_{3}$ is given by the matrices

$$
A=\left(\begin{array}{lll}
0 & x & z \\
0 & 0 & y \\
0 & 0 & 0
\end{array}\right)
$$


with $x, y, z \in \mathbb{R}$.The exponential map exp $: \mathfrak{h}_{3} \rightarrow \mathbb{H}_{3}$ is a global diffeomorphism, and is given by

$$
\exp (A)=I+A+\frac{A^{2}}{2}=\left(\begin{array}{ccc}
1 & x & z+\frac{x y}{2} \\
0 & 1 & y \\
0 & 0 & 1
\end{array}\right)
$$

The Heisenberg group $\mathbb{H}_{3}$ is represented as the cartesian 3 -space $\mathbb{R}^{3}(x, y, z)$ with group structure:

$$
\left(x_{1}, y_{1}, z_{1}\right) \cdot\left(x_{2}, y_{2}, z_{2}\right):=\left(x_{1}+x_{2}, y_{1}+y_{2}, z_{1}+z_{2}+\frac{1}{2} x_{1} y_{2}-\frac{1}{2} x_{2} y_{1}\right) .
$$

We equip $\mathbb{H}_{3}$ with the following left invariant Riemannian metric

$$
g:=d x^{2}+d y^{2}+\left(d z+\frac{1}{2}(y d x-x d y)\right)^{2} .
$$

The identity component $I^{\circ}\left(\mathbb{H}_{3}\right)$ of the full isometry group of $\left(\mathbb{H}_{3}, g\right)$ is the semi-direct product $S O(2) \ltimes \mathbb{H}_{3}$. The action of $S O(2) \ltimes \mathbb{H}_{3}$ is given explicitly by

$$
\begin{aligned}
A & =\left(\left[\begin{array}{cc}
\cos \theta & -\sin \theta \\
\sin \theta & \cos \theta
\end{array}\right] \cdot\left[\begin{array}{l}
a \\
b \\
c
\end{array}\right]\right) \cdot\left[\begin{array}{l}
x \\
y \\
z
\end{array}\right] \\
& =\left[\begin{array}{ccc}
\cos \theta & -\sin \theta & 0 \\
\sin \theta & \cos \theta & 0 \\
\frac{1}{2}(a \sin \theta-b \cos \theta) & \frac{1}{2}(a \cos \theta+b \sin \theta) & 1
\end{array}\right] \cdot\left[\begin{array}{l}
x \\
y \\
z
\end{array}\right]+\left[\begin{array}{l}
a \\
b \\
c
\end{array}\right] .
\end{aligned}
$$

In particular, rotational around the $z$-axis and translations:

$$
(x, y, z) \rightarrow(x, y, z+a), a \in \mathbb{R}
$$

along the $z$-axis are isometries of $\mathbb{H}_{3}$.

The Lie algebra $\mathfrak{h}_{3}$ of $I^{\circ}\left(\mathbb{H}_{3}\right)$ is generated by the following Killing vector fields:

$$
\begin{gathered}
F_{1}=\frac{\partial}{\partial x}+\frac{y}{2} \frac{\partial}{\partial z}, \quad F_{2}=\frac{\partial}{\partial y}-\frac{x}{2} \frac{\partial}{\partial z}, \\
F_{3}=\frac{\partial}{\partial z}, \quad F_{4}=-y \frac{\partial}{\partial x}+x \frac{\partial}{\partial y} .
\end{gathered}
$$

One can check that $F_{1}, F_{2}, F_{3}$ are infinitesimal transformations of the 1-parameter groups of isometries defined by

$$
G_{1}=\{(t, 0,0) \mid t \in \mathbb{R}\}, G_{2}=\{(0, t, 0) \mid t \in \mathbb{R}\}, G_{3}=\{(0,0, t) \mid t \in \mathbb{R}\},
$$

respectively. Here this groups acts on $\mathbb{H}_{3}$ by the left translation. The vector field $F_{4}$ generates the group of rotations around the $z$-axis. Thus $G_{4}$ is identified with $S O(2)$. 
Definition 2.1. A surface $\Sigma$ in the Heisenberg space $\mathbb{H}_{3}$ is said to be invariant surface if it is invariant under the action of the 1-parameter subgroups of isometries $\left\{G_{i}\right\}$, with $i \in\{1,2,3,4\}$.

The Lie algebra $\mathfrak{h}_{3}$ of $\mathbb{H}_{3}$ has an orthonormal basis $\left\{E_{1}, E_{2}, E_{3}\right\}$ defined by

$$
E_{1}=\frac{\partial}{\partial x}-\frac{y}{2} \frac{\partial}{\partial z}, E_{2}=\frac{\partial}{\partial y}+\frac{x}{2} \frac{\partial}{\partial z}, E_{3}=\frac{\partial}{\partial z} .
$$

The Levi-Civita connection $\nabla$ of $g$, in terms of the basis $\left\{E_{i}\right\}_{i=1,2,3}$ is explicitly given as follows

$$
\left\{\begin{array}{l}
\nabla_{E_{1}} E_{1}=0, \nabla_{E_{1}} E_{2}=\frac{1}{2} E_{3}, \nabla_{E_{1}} E_{3}=-\frac{1}{2} E_{2} \\
\nabla_{E_{2}} E_{1}=-\frac{1}{2} E_{3}, \nabla_{E_{2}} E_{2}=0, \nabla_{E_{2}} E_{3}=\frac{1}{2} E_{1} \\
\nabla_{E_{3}} E_{1}=-\frac{1}{2} E_{2}, \nabla_{E_{3}} E_{2}=\frac{1}{2} E_{1}, \nabla_{E_{3}} E_{3}=0
\end{array}\right.
$$

The Riemannian curvature tensor $R$ is a tensor field on $\mathbb{H}_{3}$ defined by

$$
R(X, Y) Z=\nabla_{X} \nabla_{Y} Z-\nabla_{Y} \nabla_{X} Z-\nabla_{[X, Y]} Z
$$

The components $\left\{R_{i j k}^{l}\right\}$ are computed as

$$
R_{212}^{1}=-\frac{3}{4}, R_{313}^{1}=\frac{1}{4}, R_{323}^{2}=\frac{1}{4} .
$$

Let us denote $K_{i j}=K\left(E_{i}, E_{j}\right)$ the sectional curvature of the plane spanned by $E_{i}$ and $E_{j}$.Then we get easily the following:

$$
K_{12}=-\frac{3}{4}, K_{13}=-\frac{1}{4}, K_{23}=-\frac{1}{4}
$$

The Ricci curvature Ric is defined by

$$
\operatorname{Ric}(X, Y)=\operatorname{trace}\{Z \rightarrow R(Z, X) Y\} .
$$

The components $\left\{R_{i j}\right\}$ of Ric are defined by

$$
\operatorname{Ric}\left(E_{i}, E_{j}\right)=R_{i j}=\sum_{k=1}^{3}\left\langle R\left(E_{i}, E_{k}\right) E_{k}, E_{j}\right\rangle .
$$

The components $\left\{R_{i j}\right\}$ are computed as

$$
R_{11}=-\frac{1}{2}, R_{12}=R_{13}=R_{23}=0, R_{22}=-\frac{1}{2}, R_{33}=\frac{1}{2} .
$$

The scalar curvature $S$ of $\mathbb{H}_{3}$ is constant and we have

$$
S=\operatorname{tr} \operatorname{Ric}=\sum_{i=1}^{3} \operatorname{Ric}\left(E_{i}, E_{i}\right)=-\frac{1}{2} .
$$




\section{Constant extrinsically Gaussian curvature $G_{1}$-invariant translation surfaces in Heisenberg group $\mathbb{H}_{3}$}

\section{1 .}

In this subsection we study complete extrinsically flat translation surfaces $\Sigma$ in Heisenberg group $\mathbb{H}_{3}$ which are invariant under the one parameter subgroup $G_{1}$. Clearly, such a surface is generated by a curve $\gamma$ in the totally geodesic plane $\{x=0\}$. Discarding the trivial case of a vertical plane $\left\{y=y_{0}\right\}$. Thus $\gamma$ is given by $\gamma(y)=(0, y, v(y))$. Therefore the generated surface is parameterized by

$$
X(x, y)=(x, 0,0) \cdot(0, y, v(y))=\left(x, y, v(y)+\frac{x y}{2}\right),(x, y) \in \mathbb{R}^{2} .
$$

We have an orthogonal pair of vector fields on $(\Sigma)$, namely,

$$
e_{1}:=X_{x}=\left(1,0, \frac{y}{2}\right)=E_{1}+y E_{3} .
$$

and

$$
e_{2}:=X_{y}=\left(0,1, v^{\prime}+\frac{x}{2}\right)=E_{2}+v^{\prime} E_{3} .
$$

The coefficients of the first fundamental form are:

$$
E=\left\langle e_{1}, e_{1}\right\rangle=1+y^{2}, F=\left\langle e_{1}, e_{2}\right\rangle=y v^{\prime}, G=\left\langle e_{2}, e\right\rangle=1+v^{\prime 2} .
$$

As a unit normal field we can take

$$
N=\frac{-y}{\sqrt{1+y^{2}+v^{\prime 2}}} E_{1}-\frac{v^{\prime}}{\sqrt{1+y^{2}+v^{\prime 2}}} E_{2}+\frac{1}{\sqrt{1+y^{2}+v^{\prime 2}}} E_{3}
$$

The covariant derivatives are

$$
\begin{gathered}
\widetilde{\nabla}_{e_{1}} e_{1}=-y E_{2} \\
\widetilde{\nabla}_{e_{1}} e_{2}=\frac{y}{2} E_{1}-\frac{v^{\prime}}{2} E_{2}+\frac{1}{2} E_{3} \\
\widetilde{\nabla}_{e_{2}} e_{2}=v^{\prime} E_{1}+v^{\prime \prime} E_{3} .
\end{gathered}
$$

The coefficients of the second fundamental form are

$$
\begin{aligned}
& l=\left\langle\widetilde{\nabla}_{e_{1}} e_{1}, N\right\rangle=\frac{y v^{\prime}}{\sqrt{1+y^{2}+v^{\prime 2}}} \\
& m=\left\langle\widetilde{\nabla}_{e_{1}} e_{2}, N\right\rangle=\frac{-\frac{y^{2}}{2}+\frac{v^{\prime 2}}{2}+\frac{1}{2}}{\sqrt{1+y^{2}+v^{\prime 2}}} \\
& n=\left\langle\widetilde{\nabla}_{e_{2}} e_{2}, N\right\rangle=\frac{-y v^{\prime}+v^{\prime \prime}}{\sqrt{1+y^{2}+v^{\prime 2}}} .
\end{aligned}
$$


Let $K_{\text {ext }}$ be the extrinsic Gauss curvature of $\Sigma$,

$$
K_{e x t}=\frac{l n-m^{2}}{E G-F^{2}}=\frac{-y^{2} v^{\prime 2}+y v^{\prime} v^{\prime \prime}-\left(-\frac{y^{2}}{2}+\frac{v^{\prime 2}}{2}+\frac{1}{2}\right)^{2}}{\left(1+y^{2}+v^{\prime 2}\right)^{2}} .
$$

Thus $\Sigma$ is extrinsically flat invariant surface in Heisenberg group $\mathbb{H}_{3}$ if and only if

$$
K_{\text {ext }}=0,
$$

that is, if and only if

$$
-y^{2} v^{\prime 2}+y v^{\prime} v^{\prime \prime}-\left(-\frac{y^{2}}{2}+\frac{v^{2}}{2}+\frac{1}{2}\right)^{2}=0
$$

to classify extrinsically flat invariant surfaces must solve the equation (3.1). We can writes equation (3.1) as

$$
y^{2}+y v^{\prime} v^{\prime \prime}-\left(\frac{y^{2}}{2}+\frac{v^{\prime 2}}{2}+\frac{1}{2}\right)^{2}=0
$$

we assume that $z=\frac{y^{2}}{2}+\frac{v^{\prime 2}}{2}+\frac{1}{2}$. Then

$$
\left\{\begin{array}{l}
z^{\prime}=y+v^{\prime} v^{\prime \prime} \\
v^{\prime} v^{\prime \prime}=z^{\prime}-y \\
v^{\prime 2}=2 z-y^{2}-1
\end{array}\right.
$$

Therefore equation (3.2) becomes

$$
y z^{\prime}-z^{2}=0
$$

equation (3.4) implies that

$$
-\frac{z^{\prime}}{z^{2}}=-\frac{1}{y}
$$

and equation (3.5) implies that

$$
z=\frac{1}{-\ln (y)+\alpha}
$$

where $\alpha \in \mathbb{R}$, and if $y \neq e^{\alpha}$.

From (3.3) and (3.6), we have

$$
\begin{aligned}
v^{\prime 2} & =2 z-y^{2}-1 \\
& =\frac{2}{-\ln (y)+\alpha}-y^{2}-1 .
\end{aligned}
$$

Thus

$$
v^{\prime}=\sqrt{\frac{2}{-\ln (y)+\alpha}-y^{2}-1} .
$$

As conclusion, we have 
Theorem 3.1. - The only non-extendable extrinsically flat translation surfaces in the 3-dimensional Heisenberg group $\mathbb{H}_{3}$ invariant under the 1-parameter subgroup $G_{1}=\left\{(t, 0,0) \in \mathbb{H}_{3} / t \in \mathbb{R}\right\}$, are the surfaces whose parametrization is $X(x, y)=$ $\left(x, y, v(y)+\frac{x y}{2}\right)$ where $y$ and $v$ satisfy

$$
v(y)=\int \sqrt{\frac{2}{-\ln (y)+\alpha}-y^{2}-1} d y .
$$

where $\alpha \in \mathbb{R}$, and $y \neq e^{\alpha}$.

- There are no complete extrinsically flat translation surfaces in the 3-dimensional Heisenberg group $\mathbb{H}_{3}$ invariant under the 1-parameter subgroup $G_{1}=\{(t, 0,0) \in$ $\left.\mathbb{H}_{3} / t \in \mathbb{R}\right\}$.

Remark 3.2. Let $\Sigma$ be a $G_{1}$-invariant translation surfaces in the 3 -dimensional Heisenberg space. Then $\Sigma$ is locally expressed as

$$
X(x, y)=(0, y, v(y)) \cdot(x, 0,0)=\left(x, y, v(y)-\frac{x y}{2}\right) .
$$

Then the extrinsically Gaussian curvature $K_{\text {ext }}$ of $\Sigma$ is computed as

$$
K_{e x t}=\frac{\left(\left(v^{\prime}-x\right)^{2}-1\right)^{2}}{4\left(1+\left(v^{\prime}-x\right)^{2}\right)^{2}} .
$$

Thus $\Sigma$ can not be of constant extrinsically Gaussian curvature.

\section{2 .}

In this subsection we study complete constant extrinsically Gaussian curvature translation surfaces $\Sigma$ in Heisenberg group $\mathbb{H}_{3}$ which are invariant under the one parameter subgroup $G_{1}$. Clearly, such a surface is generated by a curve $\gamma$ in the totally geodesic plane $\{x=0\}$. Discarding the trivial case of a vertical plane $\left\{y=y_{0}\right\}$. Thus $\gamma$ is given by $\gamma(y)=(0, y, v(y))$. Therefore the generated surface is parameterized by

$$
X(x, y)=(x, 0,0) .(0, y, v(y))=\left(x, y, v(y)+\frac{x y}{2}\right),(x, y) \in \mathbb{R}^{2} .
$$

Theorem 3.3. - The $G_{1}$-invariant constant extrinsically Gaussian curvature translation surfaces in the 3-dimensional Heisenberg group $\mathbb{H}_{3}$, are:

1. $K_{\text {ext }}=-\frac{1}{4}$.

The surfaces of equation

$$
z=v(y)+\frac{x y}{2}=\frac{x y}{2}+\frac{1}{2} y \sqrt{2 \beta-y^{2}}+\arctan \left(\frac{y}{\sqrt{\beta-y^{2}}}\right),
$$

where $\beta \in \mathbb{R}$. 
2. $K_{\text {ext }} \neq-\frac{1}{4}$.

Then $y$ and $v$ satisfy

$$
v(y)=\int \sqrt{\frac{1}{-2\left(K_{e x t}+\frac{1}{4}\right) \ln (y)+\gamma}-y^{2}-1} d y .
$$

where $\gamma \in \mathbb{R}$, and $y \neq e^{\frac{\gamma}{2\left(K_{e x t}+\frac{1}{4}\right)}}$.

- There are no complete constant extrinsically Gaussian curvature translation surfaces in the 3-dimensional Heisenberg group $\mathbb{H}_{3}$ invariant under the 1-parameter subgroup $G_{1}$.

Proof. From (4.1) and (3.2) we have

$$
K_{e x t}=\frac{l n-m^{2}}{E G-F^{2}}=\frac{y^{2}+y v^{\prime} v^{\prime \prime}-\frac{1}{4}\left(1+y^{2}+v^{\prime 2}\right)^{2}}{\left(1+y^{2}+v^{\prime 2}\right)^{2}} .
$$

1. If $K_{e x t}=-\frac{1}{4}$. Then equation (3.7) becomes

$$
y^{2}+y v^{\prime} v^{\prime \prime}=0
$$

We note that $y$ equal zero is solution of the equation(3.8).

If $y$ is different to zero $(y \neq 0)$, equation (3.8) becomes

$$
v^{\prime} v^{\prime \prime}=-y \text {. }
$$

Integration gives us

$$
v(y)=\frac{1}{2} y \sqrt{2 \beta-y^{2}}+\arctan \left(\frac{y}{\sqrt{\beta-y^{2}}}\right),
$$

where $\beta \in \mathbb{R}$.

2. If $K_{\text {ext }} \neq-\frac{1}{4}$. Then equation (3.7) becomes

$$
y^{2}+y v^{\prime} v^{\prime \prime}=\left(K_{e x t}+\frac{1}{4}\right)\left(1+y^{2}+v^{\prime 2}\right)^{2} .
$$

In fact, put $z=1+y^{2}+v^{\prime 2}$. Then $z$ satisfies

$$
\frac{1}{2} y z^{\prime}=\left(K_{e x t}+\frac{1}{4}\right) z^{2}
$$

Hence we have

$$
z=\frac{1}{-2\left(K_{e x t}+\frac{1}{4}\right) y+\gamma}
$$

where $\gamma \in \mathbb{R}$, and $y \neq e^{\frac{\gamma}{2\left(K_{e x t}+\frac{1}{4}\right)}}$. Using the equation $z=1+y^{2}+v^{\prime 2}$, we get

$$
v^{\prime 2}=\frac{1}{-2\left(K_{e x t}+\frac{1}{4}\right) y+\gamma}-y^{2}-1 .
$$




\section{Constant extrinsically Gaussian curvature $G_{2^{-}}$-invariant translation surfaces in Heisenberg group $\mathbb{H}_{3}$}

In this section we study constant complete extrinsically flat translation surfaces $\Sigma$ in Heisenberg group $\mathbb{H}_{3}$ which are invariant under the one parameter subgroup $G_{2}$. Clearly, such a surface is generated by a curve $\gamma$ in the totally geodesic plane $\{y=0\}$. Discarding the trivial case of a vertical plane $\left\{x=x_{0}\right\}$. Thus $\gamma$ is given by $\gamma(x)=(x, 0, f(x))$. Therefore the generated surface is parameterized by

$$
X(x, y)=(0, y, 0) \cdot(x, 0, f(x))=\left(x, y, f(x)-\frac{x y}{2}\right),(x, y) \in \mathbb{R}^{2} .
$$

We have an orthogonal pair of vector fields on $(\Sigma)$, namely,

$$
e_{1}:=X_{x}=\left(1,0, f^{\prime}-\frac{y}{2}\right)=E_{1}+f^{\prime} E_{3} .
$$

and

$$
e_{2}:=X_{y}=\left(0,1,-\frac{x}{2}\right)=E_{2}-x E_{3} .
$$

The coefficients of the first fundamental form are:

$$
E=\left\langle e_{1}, e_{1}\right\rangle=1+f^{\prime 2}, F=\left\langle e_{1}, e_{2}\right\rangle=-x f^{\prime}, G=\left\langle e_{2}, e\right\rangle=1+x^{2} .
$$

As a unit normal field we can take

$$
N=\frac{-f^{\prime}}{\sqrt{1+x^{2}+f^{\prime 2}}} E_{1}+\frac{x}{\sqrt{1+x^{2}+f^{\prime 2}}} E_{2}+\frac{1}{\sqrt{1+x^{2}+f^{\prime 2}}} E_{3} .
$$

The covariant derivatives are

$$
\begin{gathered}
\widetilde{\nabla}_{e_{1}} e_{1}=-f^{\prime} E_{2}+f^{\prime \prime} E_{3} \\
\widetilde{\nabla}_{e_{1}} e_{2}=\frac{f^{\prime}}{2} E_{1}+\frac{x}{2} E_{2}-\frac{1}{2} E_{3} \\
\widetilde{\nabla}_{e_{2}} e_{2}=-x E_{1} .
\end{gathered}
$$

The coefficients of the second fundamental form are

$$
\begin{aligned}
& l=\left\langle\widetilde{\nabla}_{e_{1}} e_{1}, N\right\rangle=\frac{-x f^{\prime}+f^{\prime \prime}}{\sqrt{1+x^{2}+f^{\prime 2}}} \\
& m=\left\langle\widetilde{\nabla}_{e_{1}} e_{2}, N\right\rangle=\frac{-\frac{f^{\prime 2}}{2}+\frac{x^{2}}{2}-\frac{1}{2}}{\sqrt{1+x^{2}+f^{\prime 2}}} \\
& n=\left\langle\widetilde{\nabla}_{e_{2}} e_{2}, N\right\rangle=\frac{-y v^{\prime}+v^{\prime \prime}}{\sqrt{1+y^{2}+v^{\prime 2}}} .
\end{aligned}
$$


Let $K_{\text {ext }}$ be the extrinsic Gauss curvature of $\Sigma$,

$$
K_{e x t}=\frac{\ln -m^{2}}{E G-F^{2}}=\frac{x^{2}+x f^{\prime} f^{\prime \prime}-\frac{1}{4}\left(x^{2}+f^{\prime 2}+1\right)^{2}}{\left(1+x^{2}+f^{\prime 2}\right)^{2}} .
$$

Thus $\Sigma$ is extrinsically flat invariant surface in Heisenberg group $\mathbb{H}_{3}$ if and only if

$$
K_{\text {ext }}=0,
$$

that is, if and only if

$$
x^{2}+x f^{\prime} f^{\prime \prime}-\frac{1}{4}\left(x^{2}+f^{\prime 2}+1\right)^{2}=0
$$

to classify extrinsically flat invariant surfaces must solve the equation (4.2).

We remark that the equation (4.2) is similarly to the equation (3.1), It is sufficient to change $y$ by $x$ and $v$ by $f$.

As conclusion, we have

Theorem 4.1. - The only non-extendable extrinsically flat translation surfaces in the 3-dimensional Heisenberg group $\mathbb{H}_{3}$ invariant under the 2-parameter subgroup $G_{2}=\left\{(0, t, 0) \in \mathbb{H}_{3} / t \in \mathbb{R}\right\}$, are the surfaces whose parametrization is $X(x, y)=$ $\left(x, y, f(x)-\frac{x y}{2}\right)$ where $x$ and $f$ satisfy

$$
f(x)=\int \sqrt{\frac{2}{-\ln (x)+\alpha}-x^{2}-1} d y .
$$

where $\alpha \in \mathbb{R}$, and $x \neq e^{\alpha}$.

- There are no complete extrinsically flat translation surfaces in the 3-dimensional Heisenberg group $\mathbb{H}_{3}$ invariant under the 1-parameter subgroup $G_{2}=\{(0, t, 0) \in$ $\left.\mathbb{H}_{3} / t \in \mathbb{R}\right\}$.

Remark 4.2. Let $\Sigma$ be a $G_{2}$-invariant translation surfaces in the 3 -dimensional Heisenberg space. Then $\Sigma$ is locally expressed as

$$
X(x, y)=(x, 0, f(x)) .(0, y, 0)=\left(x, y, f(x)+\frac{x y}{2}\right) .
$$

Then the extrinsically Gaussian curvature $K_{\text {ext }}$ of $\Sigma$ is computed as

$$
K_{e x t}=-\frac{\left(\left(f^{\prime}+y\right)^{2}-1\right)^{2}}{4\left(1+\left(v^{\prime}-x\right)^{2}\right)^{2}} .
$$

Thus $\Sigma$ can not be of constant extrinsically Gaussian curvature.

Theorem 4.3. - The $G_{2}$-invariant constant extrinsically Gaussian curvature translation surfaces in the 3-dimensional Heisenberg group $\mathbb{H}_{3}$, are: 
1. $K_{\text {ext }}=-\frac{1}{4}$.

The surfaces of equation

$$
z=f(x)-\frac{x y}{2}=-\frac{x y}{2}+\frac{1}{2} x \sqrt{2 \beta-x^{2}}+\arctan \left(\frac{x}{\sqrt{\beta-x^{2}}}\right),
$$

where $\beta \in \mathbb{R}$.

2. $K_{\text {ext }} \neq-\frac{1}{4}$.

Then $x$ and $f$ satisfy

$$
f(x)=\int \sqrt{\frac{1}{-2\left(K_{e x t}+\frac{1}{4}\right) \ln (x)+\gamma}-x^{2}-1} d y .
$$

where $\gamma \in \mathbb{R}$, and $x \neq e^{\frac{\gamma}{2\left(K_{\text {ext }}+\frac{1}{4}\right)}}$.

- There are no complete constant extrinsically Gaussian curvature translation surfaces in the 3-dimensional Heisenberg group $\mathbb{H}_{3}$ invariant under the 1-parameter subgroup $G_{2}$.

Acknowledgements. The author would like to thank the Referees for all helpful comments and suggestions that have improved the quality of our initial manuscript. The author would like also to thank Rabah Souam for their interest and helpful discussions and advice.

\section{References}

[1] U. Abresch, H. Rozenberg: A Hopf differential for constant mean curvature surfaces in $\mathbb{S}^{2} \times \mathbb{R}$ and $\mathbb{H}^{2} \times \mathbb{R}$, Acta Math 193 (2004), pp. 141-174.

[2] M. BekKar: Exemples de surfaces minimales dans l'espace de Heisenberg $\mathbb{H}_{3}$, Rend.Sem. Mat. Univ. Cagliari 61 (1991), pp. 123-130.

[3] M. Bekkar, T. Sari: Surfaces Minimales réglées dans l'espace de Heisenberg $\mathbb{H}_{3}$, Rend. Sem. Mat. Univ. Politec. Torino 50.3 (1992), pp. 243-254.

[4] M. Belkhelfa: Parallel and minimal surfaces in Heisenberg, in: Proceedings of the Summer School on Differential Geometry, Coimbra (Portugal), September 3-7, 1999, pp. 67-76.

[5] D. Bensikaddour, L. Belarbi: Minimal Translation Surfaces in Lorentz-Heiesenberg 3Space, Nolinear Studies 24.4 (2017), pp. 859-867.

[6] D. Bensikaddour, L. Belarbi: Minimal Translation Surfaces in Lorentz-Heiesenberg 3space with Flat Metric, Differential Geometry-Dynamical Systems 20 (2018), pp. 1-14.

[7] F. Bonahon: Geometric structures on 3-manifolds, in: In Handbook of geometric topology, North-Holland, Amsterdam, 2002, pp. 93-164.

[8] R. Cadeo, P. Piu, A. Ratto: SO(2)-invariant minimal and constant mean curvature surfaces in 3-dimensional homogeneous spaces, Maniscripta Math 87 (1995), pp. 1-12.

[9] B. Daniel: Isometric immersions into 3-dimensional homogeneous manifolds, Comment. Math. Helv 82 (2007), pp. 87-131. 
[10] B. Daniel, W. H. Meeks, H. Rosenberg: Half-space theorems for minimal surfaces in Nil and Sol, J. Differential Geometry 88 (2011), pp. 41-59.

[11] R. S. Erap, E. Toubiana: Screw motion surfaces in $\mathbb{H}^{2} \times \mathbb{R}$ and $\mathbb{S}^{2} \times \mathbb{R}$, Illinois J. Math 49 (2005), pp. 1323-1362.

[12] C. B. Figueroa, F. Mercuri, R. H. L. Pedrosa: Invariant minimal surfaces of the Heisenberg groups, Ann. Ital 7 (1999), pp. 173-194.

[13] J. Inogochi, T. Kumamoto, N. Ohsugi, Y. Suyama: Differential geometry of curves and surfaces in 3-dimensional homogeneous speces I, Fukuoka Univ. Sci. Rep 29 (1999), pp. 155182.

[14] J. Inoguchi: Flat translation surfaces in the 3-dimensional Heisenberg group, J. Geom 82.1-2 (2005), pp. 83-90, DOI: 10.1007/s00022-005-1730-1.

[15] J. Inoguchi, R. López, M. I. Munteanu: Minimal translation surfaces in the Heisenberg group $\mathrm{Nil}_{3}$, Geom. Dedicata 161 (2012), pp. 221-231, DOI: 10.1007/s10711-012-9702-8.

[16] R. López: Constant mean curvature surfaces in Sol with non-empty boundary, Houston J. Math 38.4 (2012), pp. 1091-1105.

[17] R. López, M. I. Munteanu: Invariant surfaces in homogeneous space Sol with constant curvature, Math. Nach 287.8 (2014), pp. 1013-1024, DOI: 10.1002/mana.201010083.

[18] R. López, M. I. Munteanu: Minimal translation surfaces in $\mathrm{Sol}_{3}$, J. Math. Soc. Japan 64.3 (2012), pp. 985-1003, DOI: $10.2969 /$ jms j/06430985.

[19] J. M. Manzano, R. Souam: The classification of totally ombilical surfaces in homogeneous 3-manifolds, Math. Z 279 (2015), pp. 557-576.

[20] W. S. Massey: Surfaces of Gaussian curvature zero in euclidean 3-space, Tohoku Math. J. 14.1 (1962), pp. 73-79.

[21] W. H. Meeks: Constant mean curvature spheres in Sol 3 , Amer. J. Math 135 (2013), pp. 113.

[22] W. H. Meeks, J. PÉrez: Constant mean curvature in metric Lie groups, Contemp. Math 570 (2012), pp. 25-110.

[23] W. H. Meeks, H. Rosenberg: The theory of minimal surfaces in $\mathbb{M} \times \mathbb{R}$, Comment. Math. Helv 80 (2005), pp. 811-885.

[24] E. Molnár, J. Szirmai: Symmetries in the 8 homogeneous 3-geometries, Symmetry: Culture and Science 21.1-3 (2010), pp. 87-117.

[25] B. Nelli, H. Rozenberg: Minimal surfaces in $\mathbb{H}^{2} \times \mathbb{R}$, Bull. Braz. Math. Soc 33 (2002), pp. 263-292.

[26] J. Pallagi, B. Schultz, J. Szirmai: Equidistant surfaces in Nil space, Stud. Univ. Zilina. Math. Ser 25 (2011), pp. 31-40.

[27] H. Rosenberg: Minimal surfaces in $\mathbb{M}^{2} \times \mathbb{R}$, Illinois J. Math 46 (2002), pp. 1177-1195.

[28] A. SAnini: Gauss map of surfaces of Heisenberg group, Boll. Un. Math. Ital 7.11-B, Supl. Facs. 2 (1997), pp. 79-93.

[29] P. Sсотт: The geometries of 3-manifolds, Bull. London Math. Soc 15 (1983), pp. 401-487, DOI: $10.1112 / \mathrm{blms} / 15.5 .401$.

[30] R. SouAm: On stable constant mean curvature surfaces in $\mathbb{S}^{2} \times \mathbb{R}$ and $\mathbb{H}^{2} \times \mathbb{R}$, Trans. Amer. Math. Soc 362.6 (2010), pp. 2845-2857.

[31] R. Sounm, E. Toubiana: On the classification and regularity of umbilic surfaces in homogeneous 3-manifolds, Mat. Contemp 30 (2006), pp. 201-215.

[32] R. Sounm, E. Toubiana: Totally umbilic surfaces in homogeneous 3-manifolds, Comm. Math. Helv 84 (2009), pp. 673-704. 
[33] J. Szirmai: Lattice-like translation ball packings in Nil space, Publ. Math. Debrecen 80.3-4 (2012), pp. 427-440.

[34] W. M. Thurston: Three-dimensional Geometry and Topology I, ed. by S. Levi, Princeton Math.Series, 35, 1997.

[35] F. Torralbo, F. Urbano: On the Gauss curvature of compact surfaces in homogeneous 3-manifolds, Proc. Amer. Math. Soc 138.7 (2010), pp. 2561-2567.

[36] D. W. Yoon, C. W. Lee, M. K. Karacan: Some Translation in the 3-dimensional Heisenberg group, Bull. Korean Math. Soc 50 (2013), pp. 1329-1343, DOI: 10.4134/BKMS.2013.50. 4.1329 .

[37] D. W. Yoon, J. W. LEe: Translation invariant surfaces in the 3-dimensional Heisenberg group, Bull. Iranian Math. Soc. 40 (2014), pp. 1373-1385. 